BMJ Open Gastroenterology

\title{
Choice of plastic or metal stent for patients with jaundice with pancreaticobiliary malignancy using simple clinical tools: a prospective evaluation
}

\author{
C Mel Wilcox, Hwasoon Kim, Toni Seay, Shyam Varadarajulu
}

To cite: Wilcox CM, Kim H, Seay $\mathrm{T}$, et al. Choice of plastic or metal stent for patients with jaundice with pancreaticobiliary malignancy using simple clinical tools: a prospective evaluation. BMJ Open Gastro 2015;2:e000014. doi:10.1136/bmjgast-2014000014

- Additional material is available. To view please visit the journal (http://dx.doi.org/ 10.1136/bmjgast-2014000014)

Received 11 August 2014 Revised 3 November 2014 Accepted 4 November 2014
Division of Gastroenterology and Hepatology, University of Alabama at Birmingham, Birmingham, Alabama, USA

Correspondence to Dr C Mel Wilcox; melw@uab.edu

\section{ABSTRACT}

Background and aim: Although plastic stents have been recommended for patients with pancreaticobiliary malignancy and an expected survival of less than 6 months, no study has developed criteria to assess survival which could then determine the choice of stent for biliary decompression. The aim of the study was to determine the utility of simple clinical tools in deciding whether to place a plastic or metal stent in patients with malignant obstructive jaundice.

Methods: At presentation for endoscopic retrograde cholangiopancreatography for suspected malignant distal bile duct obstruction, prospectively patients with Karnofsky score of $<80$ and/or metastatic disease to the liver underwent placement of 10-French plastic stents while patients with a Karnofsky score of $\geq 80$ underwent placement of self-expandable metal stents (SEMS). Long-term stent patency and mortality was determined.

Results: 98 patients (mean age 66.5 years; $62.2 \%$ male) were enrolled with $67(68.4 \%)$ receiving plastic stents and $31(31.6 \%)$ uncovered SEMS. Overall, patients receiving plastic stents had a median survival of 2.8 months compared with 11.6 months for metallic stents $(p<0.0001)$. Patients with a Karnofsky score $<80$ or liver metastases had very poor survival of 3.1 and 1.8 months, respectively. The overall reintervention rate was $42 \%$ for those receiving plastic stents and $19 \%$ for metallic stents.

Conclusions: The decision whether to place a plastic stent or SEMS for patients with distal malignant obstructive jaundice may be based on simple clinical tools resulting in low rates of reintervention.

\section{INTRODUCTION}

In patients with malignant obstructive jaundice, the endoscopists' major decision centres on placement of a biliary plastic or selfexpandable metal stent (SEMS). Current recommendations suggest that patients with an expected life expectancy of 6 months or

\section{Key messages}

What is already known about this subject

- Cancer of the pancreas and bile duct are highly lethal malignancies with short survival time

- Bile duct stenting for pancreatic and biliary malignancies are highly effective at relieving jaundice, but are not permanent

- Metal stents have a longer duration of efficacy than plastic stents but are much more expensive

What are the new findings

- The presence of metastatic disease predicted a very short survival

- A Karnofsky score of $<70$ predicted a very short survival

- Use of a plastic or metal stent may be better selected based upon these easy to determine metrics

How might it impact on clinical practice in the foreseeable future?

- Determination of the presence of metastatic disease is generally known to endoscopists at the time of evaluation of a patient with suspected pancreatic and biliary cancer. The Karnofsky score is very easy to determine.

- By using these factors at the time of stenting, one can possibly make a more cost effective determination of the best type of stent to use.

less should undergo placement of a plastic stent. ${ }^{12}$ A number of studies have examined prognostic factors for survival in patients with pancreaticobiliary malignancy that include clinical, laboratory, radiographic, pathological and surgical variables. ${ }^{3-8}$ However, a majority of these factors have little relevance to the endoscopist at the time of decision-making. The use of simple criteria which are widely available, easily applied and predictable could be a major advancement to assist in choosing the appropriate biliary endoprosthesis. 
Prior studies suggest that a low Karnofsky score which is easy to calculate and the presence of liver metastases, generally known at the time of evaluation, have prognostic significance. ${ }^{9-16}$ We prospectively evaluated the utility of these two clinical tools in determining the most ideal endoprosthesis for biliary decompression.

\section{METHODS}

Consecutive patients presenting with malignant obstructive jaundice and a mid or distal biliary stricture were included in this prospective observational cohort study beginning March 2008-June 2010 and with subsequent follow-up extending to 1 January 2014. Patients with hilar strictures were excluded. All patients signed informed consent both for the procedure and the study which was approved by our institutional review board (Protocol \# X08020811). The diagnosis of cancer was generally confirmed by endoscopic ultrasound guided fine-needle aspiration (EUS-FNA) at the time of evaluation unless the patient had prior biopsy-proven malignancy. Prior to endoscopic retrograde cholangiopancreatography (ERCP), Karnofsky score was calculated (see online supplementary appendix 1) and radiographic reports were reviewed to both confirm the location of the tumour as well as evaluate for hepatic or extrahepatic metastases. All patients had previously undergone abdominal CT scanning, the majority (88\%) with intravenous contrast. ERCP and biliary stenting was performed by two skilled endoscopists (CMW, SV).

Per protocol, patients with a Karnofsky score $<80$ and/ or liver metastasis underwent placement of 10-French plastic stents (Cook Endoscopy, Winston Salem, North Carolina, USA) of variable length depending on the characteristic of the stricture. Patients with a Karnofsky score of 80 or more underwent placement of $10 \mathrm{~mm}$ uncovered SEMS (Boston Scientific, Natick, Massachusetts, USA) of variable length depending on the characteristic of the stricture. Sphincterotomy was not generally performed for stent placement $(<5 \%)$. Patients were considered potential surgical candidates if they were referred by a surgeon who was planning Whipple resection or in whom at the time of initial evaluation comorbidity was limited, no vascular invasion was seen by EUS or CT, and metastases were absent; in these patients, a 10-French plastic stent was placed. These patients-termed preoperative candidates-are considered separately. Patients were excluded if a baseline CT scan was not performed prior to evaluation. Patients were also excluded if the stent type placed was not per protocol as some of the patients were specifically referred for a type of stent which may not have been according to protocol.

Following stent placement, patients were followed prospectively for a long-term until death or loss to follow-up. Patients were contacted every 4 months to determine if ERCP was repeated. All records for repeat procedures were reviewed. The use of chemotherapy and/or radiation therapy was documented.

\section{Main outcome measures}

Evaluating the utility of Karnofsky score and liver metastasis in predicting survival time, and determining the appropriate stent type for biliary decompression.

\section{Statistical analysis}

$\chi^{2}$ test and Fisher's exact test were used for each categorical variable to test their association among three groups. Since age for each group was normally distributed and had equal variance, one-way analysis of variance was conducted to test their mean difference. Kruskal-Wallis test was performed to assess whether one of three groups tends to have larger values than the others for Karnofsky score, which were not normally distributed. Cross-classifications were designed to identify potential interactions among potential predictors. The cumulative survival curve was depicted using the Kaplan-Meier method and patients alive at the last follow-up were censored. A priori planned analyses included evaluating factors independently associated with overall survival. This was performed by using univariate Cox proportional hazards regression analyses, and was conducted for the plastic and metal stent groups excluding the preoperative group. Analysis was performed using SAS software, V.9.3 (SAS Institute Inc, Cary, North Carolina, USA) and statistical difference was considered to be significant at the level of 0.05 .

\section{RESULTS}

\section{Patient characteristics}

A total of 106 patients were evaluated, meeting the inclusion criteria of the study between March 2008 and June 2010 and followed until 1 January 2014. No patient considered a candidate for the study declined enrolment. At the time of analysis, 98 patients $(92.5 \%)$ were dead and the overall survival of the cohort was poor with a median survival time of 8.7 months (IQR 2.4-19.2 months). Five patients were excluded as their physicians requested metallic stents though these patients were candidates for plastic stents, and three patients were excluded as only abdominal ultrasound was performed prior to ERCP as gallstone disease was suspected. The baseline characteristics of the final cohort of 98 patients and by the choice of stent are listed in table 1 . Consistent with our patient population, most patients were older, Caucasian and had pancreatic adenocarcinoma. EUS-FNA performed before $(60.2 \%)$ or after the ERCP confirmed a diagnosis of malignancy in all patients. A Whipple resection was performed in 21 patients $(21.4 \%)$ and $19(19.4 \%)$ received or had received chemoradiation. Twenty-four patients (preoperative group) considered surgical candidates underwent plastic stent placement preoperatively for biliary decompression and are considered separately. Median Karnofsky score in these 24 patients was 80 (range 80-100). The median survival of these 24 patients was 20.5 months (range 2-65 months) and 6 of these patients are alive. 
Table 1 Baseline characteristics

\begin{tabular}{|c|c|c|c|c|c|}
\hline & $\begin{array}{l}\text { Total } \\
\mathrm{N}=98\end{array}$ & $\begin{array}{l}\text { Metal stent } \\
\mathrm{N}=31(31.6 \%)\end{array}$ & $\begin{array}{l}\text { Plastic stent } \\
\mathrm{N}=43(43.9 \%)\end{array}$ & $\begin{array}{l}\text { Preoperative } \\
\text { plastic stent } \\
\mathrm{N}=24(24.5 \%)\end{array}$ & p Value \\
\hline $\mathrm{Age}^{*}$ & $66.5 \pm 11.2(34-88)$ & $66.4 \pm 11.3(34-87)$ & $66.8 \pm 11.5(45-88)$ & $66.0 \pm 11.1(46-84)$ & 0.9613 \\
\hline Gender, N (\%) & & & & & 0.2062 \\
\hline Female & $37(37.8)$ & $15(48.4)$ & $16(37.2)$ & $6(25)$ & \\
\hline Male & $61(62.2)$ & $16(51.6)$ & 27 (62.8) & $18(75)$ & \\
\hline Race, N (\%) & & & & & 0.2700 \\
\hline Caucasian & $74(75.5)$ & $25(80.7)$ & $29(67.4)$ & 20 (83.3) & \\
\hline African-American & $21(21.4)$ & $6(19.4)$ & $11(26.2)$ & $4(16.7)$ & \\
\hline Other & $3(3.1)$ & - & $3(7.1)$ & - & \\
\hline Diagnosis, N (\%) & & & & & $<0.0001 \dagger$ \\
\hline Pancreatic cancer & $57(56.4)$ & $26(83.9)$ & $16(37.2)$ & $13(54.2)$ & \\
\hline Cholangiocarcinoma & $17(16.8)$ & $1(3.2)$ & 8 (18.6) & 8 (33.3) & \\
\hline Other & $27(26.7) \ddagger$ & $4(12.9)$ & $19(44.2)$ & $3(12.5)$ & \\
\hline Chemoradiation, N (\%) & & & & & $0.0160 \dagger$ \\
\hline Prior & $8(8.2)$ & $1(3.2)$ & 7 (16.3) & - & \\
\hline Current & $11(11.2)$ & $4(12.9)$ & 7 (16.3) & - & \\
\hline Restent, N(\%) & 27 (27.6) & $6(19.4)$ & $18(41.9)$ & $3(12.5)$ & $0.0168 \dagger$ \\
\hline $\begin{array}{l}\text { Number of restenting } \\
\text { session, } N(\%)\end{array}$ & & & & & 0.1639 \\
\hline 1 & $21(21.4)$ & $6(19.4)$ & $12(27.9)$ & $3(12.5)$ & \\
\hline 2 & $3(3.1)$ & - & $3(7.0)$ & - & \\
\hline$\geq 3$ & $3(3.1)$ & - & $3(7.0)$ & - & \\
\hline Karnofsky score§ & $80(20-100)$ & $80(80-100)$ & $65(20-80)$ & $80(80-100)$ & $<0.0001 \dagger$ \\
\hline Liver metastases, N (\%) & $16(16.3)$ & - & $16(37.2)$ & - & $<0.0001 \dagger$ \\
\hline Other metastases, N (\%) & $18(18.4)$ & $3(9.7)$ & $15(35.7)$ & - & $0.0019 \dagger$ \\
\hline Whipple resection, $\mathrm{N}(\%)$ & $21(21.4)$ & $5(16.1)$ & - & $16(66.7)$ & $<0.0001 \dagger$ \\
\hline Death, N (\%) & $90(91.8)$ & 29 (93.6) & $43(100)$ & $18(75)$ & $0.0015 \dagger$ \\
\hline
\end{tabular}

\section{Survival time}

The overall mean follow-up time for the cohort of 98 patients was 13.6 months (SEMS, range $0.5-55.5$ months;

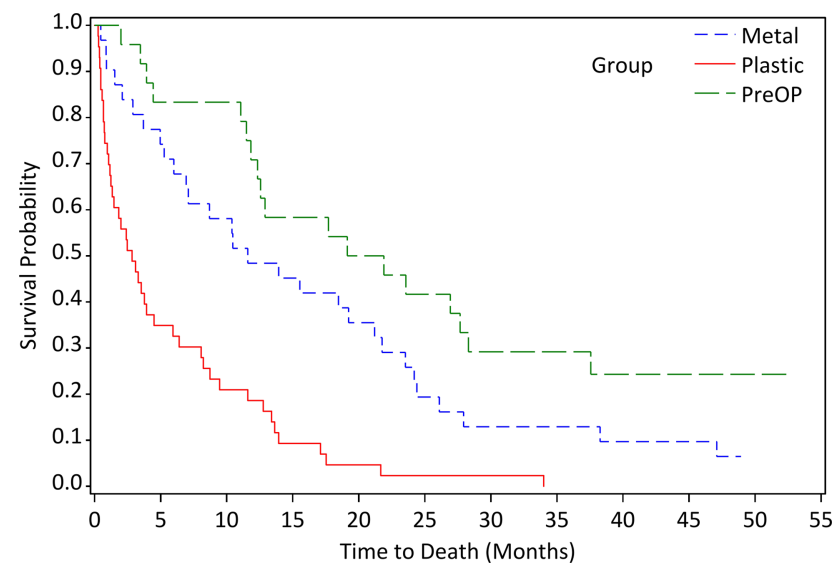

Figure 1 Long-term survival based on study groups (metal: $n=31$, median survival 11.6 months; plastic: $n=43$, median survival 2.8 months; preOP: $n=24$, median survival 20.5 months; log rank $\chi^{2}=36.0, p<0.0001$; preOP, preoperative). plastic stents, range $0.3-34$ months; plastic stents preoperatively, range 2-65.2 months). As shown in figure 1, long-term survival among the three groups was

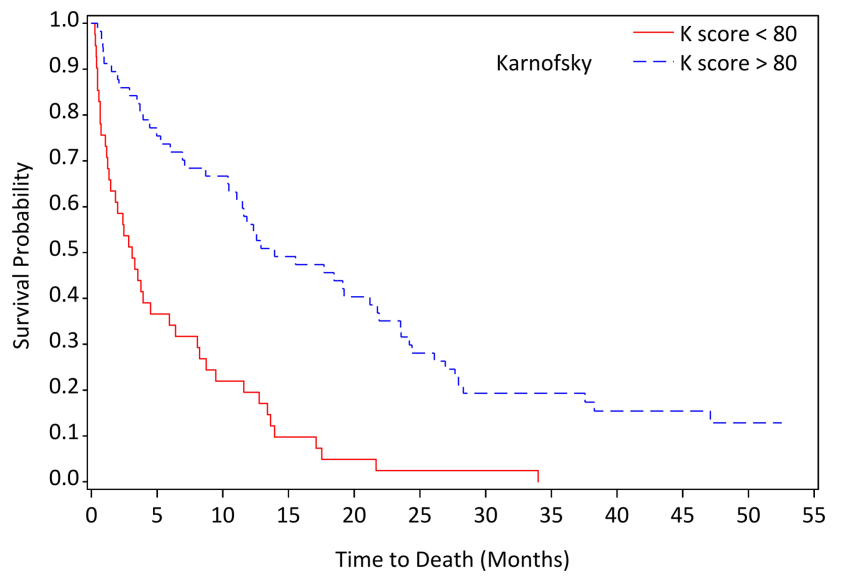

Figure 2 Long-term survival based on Karnofsky score (K score $\geq 80$ : $n=57$, median survival 13.9 months; $K$ score $<80$ : $\mathrm{n}=41$, median survival 3.1 months; log rank $\chi^{2}=28.7$, $\mathrm{p}<0.0001)$. 


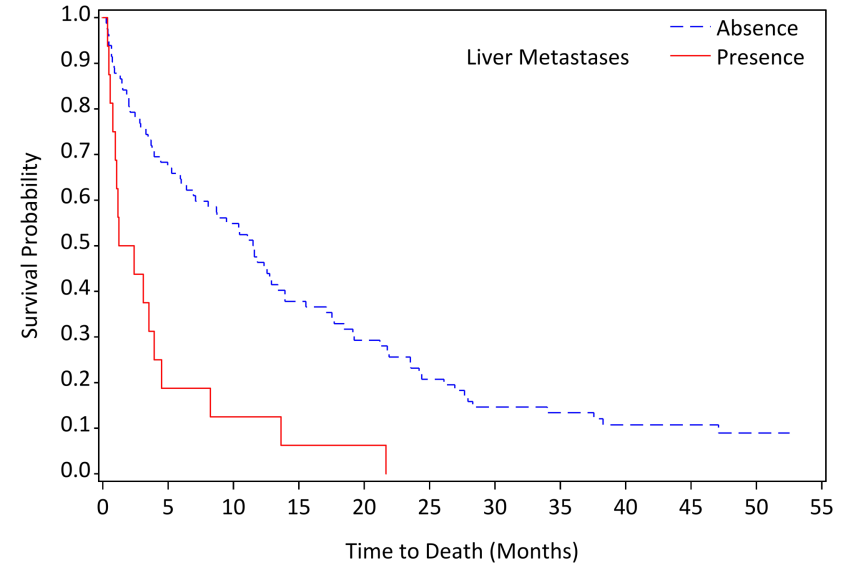

Figure 3 Long-term survival based on presence of liver metastases (absence: $\mathrm{n}=82$, median survival 11.6 months; presence: $\mathrm{n}=16$, median survival 1.8 months; log rank $\left.\chi^{2}=17.1, p<0.0001\right)$.

significantly different $\left(\log \operatorname{rank} \chi^{2}=36, p<0.0001\right)$. In figure 2, a Karnofsky score $<80$ was predictive of a shortened survival (median 3.1 months) compared with those with a score $\geq 80$ (median 13.9 months; log rank $\left.\chi^{2}=28.7, \mathrm{p}<0.0001\right)$. Likewise, patients with liver metastases (figure 3) had a shorter survival (median 1.8 months) compared with those without liver metastases (median 11.6 months; log rank $\chi^{2}=17.1, \mathrm{p}<0.0001$ ). Metastases to organs other than the liver were noted in 18 patients in whom 3 (all with a Karnofsky score of $\geq 80$ ) underwent SEMS (median survival 2.1 months) and 15 plastic stenting (median survival 3.3 months); the median survival for these 18 patients was 2.7 months (range 0.5-21.7). Patients who had a Whipple resection had a significantly longer survival (median 21.9 months) compared with those who did not have the surgery (median 5.3 months; $\log$ rank $\chi^{2}=19.5, \quad \mathrm{p}<0.0001$ ). Patients who did not receive chemoradiation had a prolonged survival compared with patients who received treatment $\left(\log\right.$ rank $\left.\chi^{2}=12.9, p=0.0003\right)$. Only two patients in the entire cohort were lost to follow-up; one underwent Whipple resection (follow-up till 49 months), and the other a plastic stent (follow-up till 36 months).

Of the 24 patients undergoing plastic stent placement 'preoperatively', 16 of these patients $(66.7 \%)$ ultimately underwent Whipple resection. Of the remaining eight patients, the median survival was 19.9 months (range 2-37.6).

\section{Stent patency}

Of these 98 patients, 67 underwent plastic stent placement for a low Karnofsky score and/or liver metastases, and 31 SEMS placements. The overall reintervention rate for these three groups was $27.5 \%(\mathrm{n}=27)$ and $41.9 \%$ $(n=18)$ for the plastic stent group; $19.4 \%(n=6)$ for the SEMS cohorts; and $12.5 \%(\mathrm{n}=3)$ for the preoperative plastic stent group (table 1). No patient with pancreatic cancer and liver metastases underwent restenting. No patient with a Karnofsky score $<50$ underwent restenting. The majority of patients with liver metastasis in whom plastic stents were placed had non-pancreatic malignancies and metastasis from other organs, such as colon or lung. In all but three patients, the patients for restenting presented with recurrent jaundice or abnormal liver tests. No patient died from stent occlusion.

\section{Univariate analysis}

In univariate Cox proportional hazards regression analyses, the patient's age, dichotomised Karnofsky score ( $\geq 80$ vs $<80$ ), presence of liver metastases, stent type, chemotherapy or radiation therapy, Whipple resection, reintervention, and number of restent were tested to evaluate an association with survival. The univariate analysis showed that patients with higher Karnofsky score or with metal stent placed or who underwent Whipple resection were associated with longer survival, but presence of liver metastases and history of chemoradiation therapy were negatively related with long-term survival (table 2).

\section{DISCUSSION}

The choice of a plastic stent or SEMS for biliary decompression in malignant obstructive jaundice is predominantly based on the endoscopist's preference. We have shown that by using simple clinical tools, a potentially better choice can be made regarding which type of endoprosthesis to deploy. For those in whom longer survival was predicted and a SEMS placed, reintervention

Table 2 Univariate analysis of prognostic factors for overall survival in metal and plastic stent group

\begin{tabular}{lllr}
\hline Predictor & HR & 95\% Cl for HR & p Value \\
\hline Age & 1.01 & $(0.99$ to 1.04$)$ & 0.1671 \\
Karnofsky score ( $\geq 80$ vs $<80)$ & 0.40 & $(0.25$ to 0.67$)$ & $0.0004^{*}$ \\
Liver metastases (yes vs no) & 2.36 & $(1.32$ to 4.22$)$ & $0.0037^{*}$ \\
Stent type (metal vs plastic) & 0.36 & $(0.22$ to 0.60$)$ & $<0.0001^{*}$ \\
Whipple resection (yes vs no) & 0.19 & $(0.06$ to 0.61$)$ & $0.0053^{*}$ \\
Chemoradiation (yes vs no) & 1.89 & $(0.65$ to 1.78$)$ & $0.0221^{*}$ \\
Restent (yes vs no) & 1.08 & $(0.80$ to 1.42$)$ & 0.7640 \\
Number of restent & 1.06 & &
\end{tabular}


was required in only six patients $(19.4 \%)$. These findings are consistent with the rate of reinterventions in selected groups reported in prior studies in patients with pancreaticobiliary malignancy. ${ }^{17} 18$

To the best of our knowledge, there are no studies that specifically address the determination of which endoprosthesis to use at the time of initial evaluation. Many prior studies have demonstrated the longer patency rates of SEMS as compared with plastic stents. Generally, metal stents remain patent for a mean of 812 months compared with 3-6 months for large plastic stents. ${ }^{17} 1920$ While the poor survival of patients with pancreaticobiliary malignancy is well recognised, there are some patients who have had a more prolonged survival and some patients who undergo Whipple resection. Given the cost of SEMS, appropriate use in those in whom surgery would not be likely or when an extended survival is predicted is appropriate. The use of plastic stents has been recommended for use in those with distant metastasis as well. ${ }^{17}$ In our cohort of 18 patients with metastases to organs other than the liver, median survival was poor. Three of these patients underwent metal stent placement because of a high Karnofsky score and the range of survival for these three patients was 0.9-11.6 months.

As noted above, a variety of prognostic factors have been studied for pancreaticobiliary malignancy, generally pancreatic cancer. Many of these factors are either not available at the time of evaluation or would not be easy to use for the endoscopist. We elected to evaluate the Karnofsky score as it is easy to calculate (see online supplementary appendix 1) and widely available at the time of evaluation. Confirming prior observations, ${ }^{9-12}$ we found that those with a low Karnofsky score were more likely to have a shortened survival with an acceptable reintervention rate even with 10-French plastic stent placement. Conversely, those in whom longer survival was predicted by the Karnofsky score and in whom a SEMS was placed, survival was longer and reintervention low. While a low Karnofsky score was helpful, it appears that in our cohort the presence of liver metastases often was as strong or a better predictor for poor mortality regardless of the Karnofsky score. Indeed, we actually evaluated three groups: higher $\mathrm{K}$ and without liver metastasis $(n=31)$, lower Karnofsky score with liver metastasis $(n=14)$, and others who have either $(n=29)$. The median survival time for three groups showed that survival for the metal group was 11.6 months and 2.8 months for the other two groups. Thus, our protocol estimated survival time quite precisely based on the recommended guideline. Liver metastases also predicted a shortened survival, as has been shown previously for pancreatic cancer. ${ }^{13-16}{ }^{21}$ Overall, however, our reintervention rate was higher than expected. We believe this relates to the heterogeneity of the patients studied which included 'all comers' as seen in daily practice with malignant obstructive jaundice. Such heterogeneity is also a limitation of the study design. Patient undergoing stenting for nodal obstruction due to metastatic disease seemed to fare better than pancreatic cancer and accounted for the higher than expected rate of repeat stenting. These findings suggest that perhaps use of the Karnofsky score or presence of liver metastases may not be as accurate for other cancers as with pancreatic cancer.

Several limitations of our study deserve mention. First, although all patients underwent CT scanning, some did not receive intravenous contrast or a three-phase scan which could possibly have improved the detection of metastases. Similarly, all patients who were potential surgical candidates were not evaluated by an experienced surgeon such that one could definitively say whether the patient would undergo surgery. Nevertheless, these are the challenges facing endoscopists with such patients and we studied the use of Karnofsky score and liver metastasis as a way to decide which prosthesis to place. Also, the majority of patients without metastatic disease had EUS prior to ERCP to examine for vascular invasion and confirm the diagnosis of pancreaticobiliary malignancy prior to ERCP. Regardless, again these limitations are what endoscopists face in real time and thus, are most realistic for routine clinical practice. Our data is germane for those with distal and not hilar strictures. Importantly, in our study, patients were followed prospectively, all records reviewed and the majority followed up at our medical centre. Finally, the use of neoadjuvant therapy for pancreatic cancer is increasing and could impact long-term survival potentially warranting the use of SEMS rather than a plastic stent.

On the basis of the data from our study, a potential approach to the choice of plastic or metal stent in patients with pancreaticobiliary malignancy can be offered. The first decision point should be the presence or absence of liver metastasis. For those with liver metastasis, consideration should be given to deployment of a plastic stent when the patient has pancreatic cancer or if the Karnofsky score is very low (50 or less). For those patients who do not have liver metastases and the Karnofsky score is high, SEMS is appropriate. While in our study we prospectively placed 10-French plastic stents in patients who were considered preoperative candidates, $66.7 \%$ of these patients ultimately went to surgery suggesting that plastic stenting was appropriate. Some patients will not undergo surgery and unless immediately scheduled for surgery or a surgical opinion has not been offered, an SEMS may be most appropriate. Emerging data ${ }^{20} 22$ suggests that SEMS may be appropriate for those who may receive neoadjuvant therapy and these are patients who are likely to have a good performance status. As our therapies for pancreatic cancer improve, the endoscopic approach to stenting will likely need to be revisited.

In summary, our study suggests that use of Karnofsky score and presence of liver or extrahepatic metastasis may potentially be beneficial in selecting the choice of a plastic or metal stent for patients with pancreaticobiliary 
malignancy. Further studies should evaluate additional markers to better define the appropriate stent as well as for evaluating the role of Karnofsky score and presence of liver metastasis in homogeneous cohorts. Prospective randomised trials also would appear relevant based on our study. Nevertheless, as chemoradiation treatments improve, it is likely that many of these inoperable patients may have a more prolonged survival and thus, further evaluation as to the appropriateness of metal stent placement and predictors for long-term outcome deserve careful study.

Contributors CMW and SV contributed in the study conception and design. CMW, TS and SV participated in the acquisition of data. CMW, HK and SV performed the analysis and interpretation of data. All the authors were involved in the drafting of manuscript and contributed to its critical revision.

Funding A portion of the study was supported by an $\mathrm{NIH}$ grant: K24 DK070629.

\section{Competing interests None.}

Provenance and peer review Not commissioned; externally peer reviewed.

Data sharing statement No additional data are available.

Open Access This is an Open Access article distributed in accordance with the Creative Commons Attribution Non Commercial (CC BY-NC 4.0) license, which permits others to distribute, remix, adapt, build upon this work noncommercially, and license their derivative works on different terms, provided the original work is properly cited and the use is non-commercial. See: http:// creativecommons.org/licenses/by-nc/4.0/

\section{REFERENCES}

1. Moss AC, Morris E, MacMathuna P. Palliative biliary stents for obstructing pancreatic carcinoma. Cochrane Database Syst Rev 2006;(2):CD004200.

2. Dumonceau JM, Tringali A, Blero D, et al. Biliary stenting: indications, choice of stents and results: European Society of Gastrointestinal Endoscopy (ESGE) clinical guideline. Endoscopy 2012;44:277-98.

3. Eloubeidi MA, Desmond RA, Wilcox CM, et al. Prognostic factors for survival in pancreatic cancer: a population-based study. Am J Surg 2006;192:322-9.

4. Tas F, Aykan F, Alici S, et al. Prognostic factors in pancreatic carcinoma: serum LDH levels predict survival in metastatic disease. Am J Clin Oncol 2001;24:547-50.
5. Humphris JL, Chang DK, Johns AL, et al. The prognostic and predictive value of serum CA 19.9 in pancreatic cancer. Ann Oncol 2012;23:1713-22.

6. Zhang DX, Dai YD, Yuan SX, et al. Prognostic factors in patients with pancreatic cancer. Exp Ther Med 2012;3:423-32.

7. La Torre M, Nigri G, Cavallini M, et al. The Glasgow Prognostic Score as a predictor of survival in patients with potentially resectable pancreatic adenocarcinoma. Ann Surg Oncol 2012;19:2917-23.

8. Prat $\mathrm{F}$, Chapat $\mathrm{O}$, Ducot $\mathrm{B}$, et al. Predictive factors for survival of patients with inoperable malignant distal biliary strictures: a practical management guideline. Gut 1998;42:76-80.

9. Maréchal R, Demols A, Gay F, et al. Prognostic factors and prognostic index for chemonaïve and gemcitabine-refractory patients with advanced pancreatic cancer. Oncology 2007;73:41-51.

10. Krishnan S, Rana V, Janjan NA, et al. Prognostic factors in patients with unresectable locally advanced pancreatic adenocarcinoma treated with chemoradiation. Cancer 2006;107:2589-96.

11. Boeck S, Hinke A, Wilkowski R, et al. Importance of performance status for treatment outcome in advanced pancreatic cancer. World $J$ Gastroenterol 2007:13:224-7.

12. Tas F, Sen F, Odabas $\mathrm{H}$, et al. Performance status of patients is the major prognostic factor at all stages of pancreatic cancer. Int $\mathrm{J}$ Clin Oncol 2013;18:839-46.

13. Weber A, Kehl V, Mittermeyer T, et al. Prognostic factors for survival in patients with unresectable pancreatic cancer. Pancreas 2010;39:1247-53.

14. Ridwelski K, Meyer F, Ebert M, et al. Prognostic parameters determining survival in pancreatic carcinoma and, in particular, after palliative treatment. Dig Dis 2001;19:85-92.

15. Neuzillet C, Sauvanet A, Hammel P. Prognostic factors for resectable pancreatic adenocarcinoma. J Visceral Surg 2011;148:e232-43.

16. Müller MW, Friess $\mathrm{H}$, Köninger $\mathrm{J}$, et al. Factors influencing survival after bypass procedures in patients with advanced pancreatic adenocarcinomas. Am J Surg 2008;195:221-8.

17. Soderlund C, Linder S. Covered metal versus plastic stents for malignant common bile duct stenosis: a prospective, randomized, controlled trial. Gastrointest Endosc 2006;63:986-95.

18. Moses PL, AINaamani KM, Barkun AN, et al. Randomized trial in malignant biliary obstruction: plastic vs partially covered metal stents. World J Gastroenterol 2013;19:8638-46.

19. Almadi MA, Barkun AN, Martel M. No benefit of covered vs uncovered self-expandable metal stents in patients with malignant distal biliary obstruction: a meta-analysis. Clin Gastroenterol Hepatol 2013;11:27-37.

20. Adams MA, Anderson MA, Myles JD, et al. Self-expanding metal stents (SEMS) provide superior outcomes compared to plastic stents for pancreatic cancer patients undergoing neoadjuvant therapy. $J$ Gastrointest Oncol 2012;3:309-13.

21. Yi JH, Lee J, Park SH, et al. A prognostic model to predict clinical outcomes with first-line gemcitabine-based chemotherapy in advanced pancreatic cancer. Oncology 2011;80:175-80.

22. Aadam AA, Evans DB, Khan A, et al. Efficacy and safety of self-expandable metal stents for biliary decompression in patients receiving neoadjuvant therapy for pancreatic cancer: a prospective study. Gastrointest Endosc 2012;76:67-75. 